\title{
Drug Development Perspectives: Considerations, Challenges and Strategies
}

\author{
"I found this book to be a unique set of examples that clearly articulate \\ many of the dilemmas researchers in the field face when working to bring \\ new medicines to the patient."
}

Over the past century, the pharmaceutical industry has been challenged by both social and economic pressures to optimize the drug-discovery process in order to improve the efficiency by which new drugs are discovered and developed. This endeavor has cultivated a large pharmaceutical industry whose individual members have employed a variety of differing strategies centered on one goal: rapid delivery of medications to the patient. Central to this enterprise has been establishing a link between the in vitro potency, physicochemical properties and absorption, distribution, metabolism, excretion and toxicity (ADME/T) characteristics of a drug candidate. While understanding the connectivity between in vitro potency and physicochemical properties provides for its own challenges, ADME/T is often cited as a major contributing factor in the failure of drug development [1]. As a result, a number of citations in the literature exist that have tried to articulate the various approaches to be considered $[2,3]$.

Drug Development Perspectives is a rather interesting collection of examples that provide the reader with a variety of case examples illustrating the dilemma existing in industry today. The author, Nuggehally Srinivas, is the Chief Scientific Officer for Vanthys Pharmaceutical Development located in Bangalore, India. Over the past 20 years, he has established himself as an accomplished clinical pharmacologist with numerous publications highlighting his career achievements. The book is divided into five parts and covers ADME-related issues surrounding development associated with bioanalysis, allometry, CYP-mediated metabolism and associated genetic polymorphisms, as well as pharmacokinetic modeling and simulations.

Chapter 1 starts most appropriately with the bioanalytical challenges facing drug developers today. Using halofantrine, an effective treatment used to treat malaria, this chapter explores many of the concerns associated with the quantitation of analytes, such as extraction, chromatography, validation, and the relationship these parameters play in understanding the preclinical/clinical results. Here, this case example, provides a backdrop where an appreciation of the issues between analyte quantitation and lymphatic transport, stereochemical metabolism and drug-drug interactions (DDIs) work together to reveal the concerns of cardiotoxicity and variable absorption. From this, a general strategy is then more thoroughly discussed, focusing in on some key considerations for bioanalytical method development, including species concerns in both discovery and development.

The idea of correlating pharmacokinetic parameters, such as clearance, volume of distribution and elimination half life, by using a power function to relate the species body weight to a predicted human parameter is at the heart of allometry and is the sole focus of the second chapter. While many challenges exist in correlating these body weight differences from individual animal species, allometry has quickly become a useful tool in guiding dose selection and escalation in clinical studies. Allometry approaches can range from a simple, singlespecies extrapolation to a much more complex, multispecies integration, which leads to questions around incorporation of species variability, metabolic differences, differences in protein binding between species and aging. These various concerns are discussed and illustrated in case example evaluations with drugs such as difloxacin, verapamil and linezolid. While allometry has found its utility, other methodologies such as physiological-based pharmacokinetic (PBPK) modeling are available, although are not discussed thoroughly in this book [4].

Cytochrome P450 enzymes represent a very large and diverse group of catalysts thought to be responsible for approximately $75 \%$ of xenobiotic drug metabolism. While CYP3A4/3A5 has been one of the major isoform(s) responsible for roughly half of xenobiotic drug metabolism, more recent evidence has demonstrated a unique role for CYP2B6 regarding polymorphic variants, inducibility and the effects on clinical

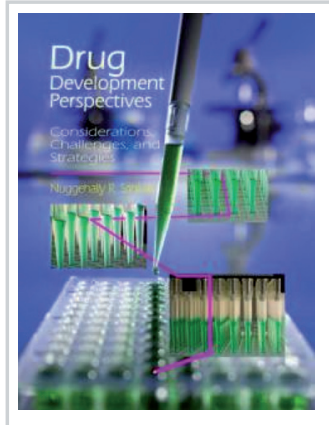

Drug Development Perspectives: Considerations, Challenges and Strategies

Author: Nuggehally R Srinivas Publisher: HNB Publishing ISBN: 978-0-9664286-9-8

\section{Elizabeth Joshi}

Lilly Research Laboratories, Department of Drug Disposition, IN 46285, USA

Tel.: +I 3176556154

Fax: + I 3174336432

E-mail: joshiem@lilly.com 
PK. A great deal of literature has been published citing the role of CYP2B6 in the stereoselective metabolism across a variety of substrates, providing the focus for Chapter 3. A number of marketed compounds are exemplified including inducers (metamizole, efavirenz, ritonavir, baicalin and atorvastatin) and inhibitors (clopidogrel, ticlopidine, mifespristone, thioTEPA and voriconazole), with an emphasis on illustrating the clinical and therapeutic challenges associated with inhibition/induction. In this chapter, readers get a general overview of the clinical drug-drug interaction study design(s) associated with each of the examples, as well as a composite list of useful in vitro and in vivo probe substrates.

The final two sections allow the reader to obtain insight into the author's perspective from a review through case studies, where a reflection of the PK and clinical/regulatory challenges facing the drug-discovery/development paradigm is discussed. Chapter 4 reflects the more modern approach to understanding a molecule's PK disposition and modulating the clinical pharmacology attributes in order to gain therapeutic benefit. Using a fluoroquinolone antibiotic widely studied in veterinarian medicine, orbifloxacin is discussed in relation to how researchers can use PK simulations in order to demonstrate utility for subjects, in this case rabbits. This question is not unlike those faced by drug developers as they look to explore problems associated with drug safety and efficacy in man. In addition, other issues surrounding drug development, such as interactions with transporters (P-glycoprotein, human organic anion transporter 3 and BCRP), practical concerns surrounding clinical pharmacology and the regulatory challenges these present are briefly reviewed as part of this chapter. The final chapter delves more deeply into the issues outlined for CYP2B6 polymorphic metabolism. Using pactimibe, rupatadine and rabeprazole as case studies, the questions of patient safety and intended efficacy are examimed as they relate to the compounds clinical drug-drug interaction concerns (inhibitor/inducer/transport) and genetic polymorphic differences in metabolism.

I found this book to be a unique set of examples that clearly articulate many of the dilemmas researchers in the field face when working to bring new medicines to the patient. There are some clear overlaps between the different chapters, especially between the DDI and metabolism concerns; however, I found these useful by highlighting the numerous, integrated facets associated with drug development. For people new to the environment, this book works to provide a deep understanding of the challenges present for both industry and academia.

Financial \& competing interests disclosure The author is employed by Eli Lilly and Company, a pharmaceutical company that discovers, development, and markets innovative drugs that save and improve lives. Lilly and Jubilant Organosys Ltd established a joint venture, Vanthys Pharmaceuticals, for early stage drug development in 2008, and Lilly still owns its interest in Vanthys. The author has no other relevant affliations or financial involvement with any organization or entity with a financial interest in or financial conflict with the subject matter or materials discussed in the manuscript apart from those disclosed.

No writing assistance was utilized in the production of this manuscript.

\section{Bibliography}

1 Li AP. Screening for human ADME/Tox drug properties in drug discovery. Drug Discovery Today 6(7), 357-366 (2001).

2 Eddershaw PJ, Beresford AP, Bayliss MK. ADME/PK as a part of a rational approach to drug discovery. Drug Discov. Today 5(9), 436-444 (2000).

3 Balani SK, Miwa GT, Gan LS, Wu JT, Lee FW. Strategy of utilizing in vitro and in vivo ADME tools for lead optimization and drug candidate selection. Curr. Top. Med. Chem. 5(11), 1033-1038 (2005).

4 Nestorov I. Whole-body physiologically based pharmacokinetic models. Expert Opin. Drug Metab. Toxicol. 3(2), 235-249 (2007). 